\title{
Difficult tracheostomy tube placement to neck breather with chronic stenosis after total laryngectomy
}

\author{
Dongseok Kim, Samsoon Cho \\ Department of Anesthesiology and Pain Medicine, VHS Medical Center, Seoul, Korea
}

Received September 2, 2021

Revised October 25, 2021

Accepted October 28, 2021

Corresponding author

Samsoon Cho

Department of Anesthesiology and Pain Medicine, VHS Medical

Center, 53 Jinhwangdo-ro 61-gil,

Gangdong-gu, Seoul 05368, Korea

Tel: +82-2-2225-1473

Fax: +82-2-2225-1946

E-mail: chss1756@naver.com

ORCID:

https://orcid.org/0000-0003-4039-8140

\begin{abstract}
As neck breathers have been proven to improve life expectancy, the chance of requiring neck breathing treatment is increasing. A 75-year-old man with a history of total laryngectomy in 1995 for laryngeal cancer was diagnosed with mechanical obstruction due to colorectal cancer. Emergency Hartmann's operation was performed. We pre-evaluated a narrow stoma airway measuring $5 \mathrm{~mm}$ with chronic stenosis. Anesthesia was successfully induced using a $4.5-\mathrm{mm}$ tube with a fiberoptic scope. After stomaplasty, we converted to a 7.0-mm tube. Moreover, we pre-evaluated the neck breather's airway, and planned how to induce anesthesia and cope with emergency situations.
\end{abstract}

Keywords: Difficult airway; Fiberoptic guided intubation; Neck breather; Stoma complication; Tracheostomy intubation

\section{INTRODUCTION}

In 2018, the number of patients with laryngeal cancer in Korea was 11,416 ( $0.6 \%$ of all patients with cancer), with a 5-year survival rate of 78.6\%. In 2020, 873 patients underwent airway surgery, such as cordectomy, partial laryngectomy, and total laryngectomy [1]. With an increased life expectancy of patients with laryngeal cancer, there has also been an increase in the number of surgeries performed under general anesthesia for patients who have permanent tracheostomy after laryngectomy.

Patients with a history of tracheostomy or head and neck surgery are expected by anesthesiologists to have a difficult airway. Neck breathers' airways require careful attention during anesthesia, as it can be affected by many complica- tions. Although patients with tracheostomy or no history of laryngectomy can breathe independently, it can be a major challenge during intubation, as there complications during wound healing or chemoradiation therapy may occur. In total laryngectomy, the airway from the oropharynx to the trachea becomes completely blocked, which makes permanent tracheostomy the only available airway. Tracheal stomas may pose a challenge during intubation due to complications, including stoma stenosis and tissue granulation.

In this case study, we successfully induced anesthesia on a patient with a neck breather, who had a deformed airway due to complications, as an emergency operation. Based on this case, we would like to discuss the difficulties associated with airway management of neck-breathing patients. 


\section{CASE REPORT}

A 75-year-old man (weight $59.2 \mathrm{~kg}$, height $170 \mathrm{~cm}$, body mass index $20.5 \mathrm{~kg} / \mathrm{m}^{2}$ ) was admitted due to constipation and abdominal distension, which started ten days prior. Following the diagnosis of mechanical obstruction related to colorectal cancer, the patient was admitted for emergency Hartmann's operation. His initial vital signs before anesthesia were as follows: blood pressure, $138 / 80 \mathrm{mmHg}$; heart rate, 99 beats/min; peripheral oxygen saturation $\left(\mathrm{SpO}_{2}\right)$, 92\%; and the patient was alert and oriented.

The patient had a history of total laryngectomy in 1995 for laryngeal cancer, and was breathing independently through a permanent tracheal stoma. He had difficulty breathing due to chronic stenosis of the stoma, but was not under any active treatment. Arterial blood gas analysis revealed a par-

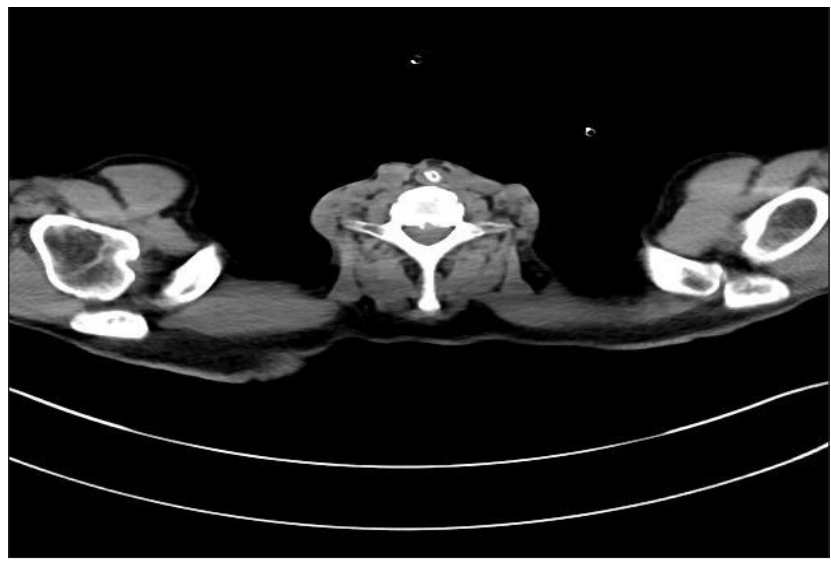

Fig. 1. Airway space is not visible at the C7 level, and only the L-tube in the esophagus is identified.

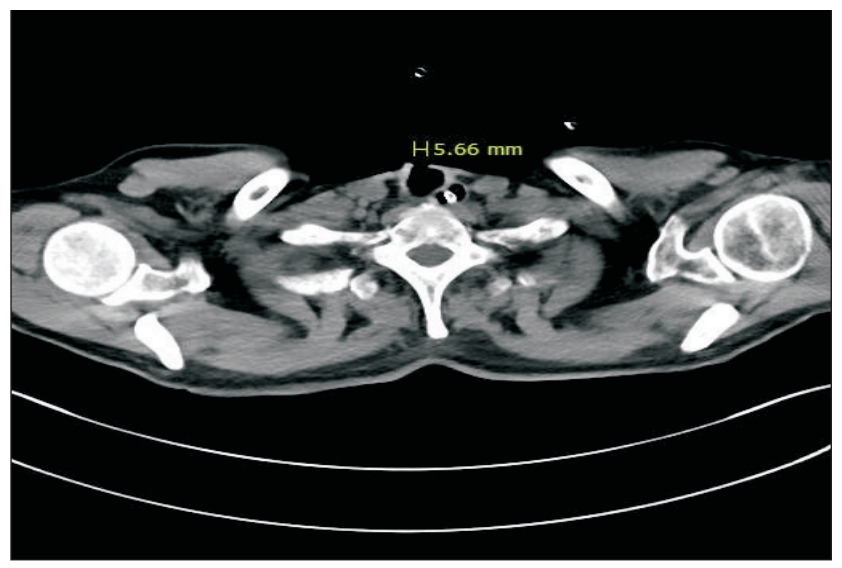

Fig. 2. A 5.66-mm outer diameter of a tracheal stoma is identified at the C7 level. tial pressure of oxygen and $\mathrm{SpO}_{2}$ of $105 \mathrm{mmHg}$ and $97 \%$ in room air, respectively.

Stomal opening was evaluated before surgery. Computed tomography (CT) imaging (Fig. 1) and laryngoscopy, combined with evaluation by an otorhinolaryngologist, confirmed that there was no airway accessible from the oropharynx. The outer diameter of the tracheal stoma was only 5-6 mm due to chronic stenosis (Fig. 2). The straightline distance from the $\mathrm{C} 7$ level, where the stoma is located at the carina, was approximately $82 \mathrm{~mm}$ (Fig. 3). CT imaging clearly depicted tracheal stenosis and granulation inside the trachea. The stoma opening was thickened due to fibrosis caused by chronic stenosis (Fig. 4). A cuffed endotracheal tube (ID $4.5 \mathrm{~mm} / \mathrm{OD} 6.2 \mathrm{~mm}$, Mallinckrodt ${ }^{\mathrm{TM}}$, COVIDIEN ${ }^{\mathrm{TM}}$ ) was able to pass through the stoma. We confirmed that a fiberoptic scope $(3.7 \mathrm{~mm}$ in diameter, Storz fiberscope, Germany) could also pass through the $4.5-\mathrm{mm}$ endotracheal tube (ETT), since high lung positive pressure was expected to maintain ventilation in this patient with severe bowel distention, and the patient complained of difficulties in self-respiration through a narrow tracheal stoma. Therefore, we decided to initiate stomaplasty prior to Hartmann's operation. The possibility of local stomaplasty was discussed by an otorhinolaryngologist prior to intubation; however, the surgery was performed under general anesthesia, as

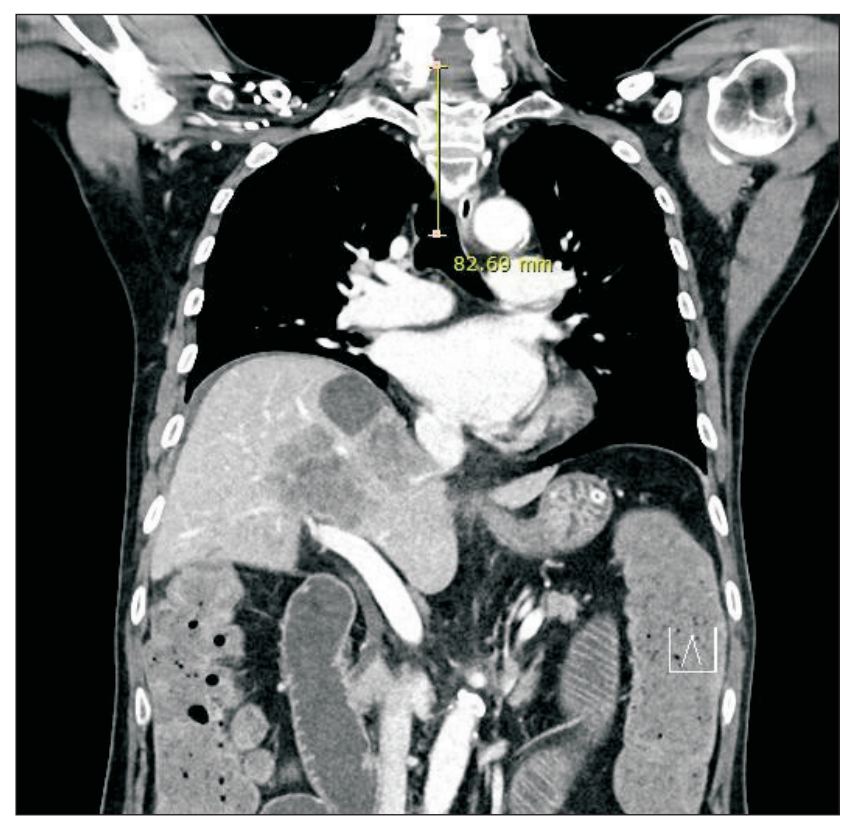

Fig. 3. Confirmed approximately $82 \mathrm{~mm}$ of a straight line distance from the $\mathrm{C} 7$ body to the carina. 


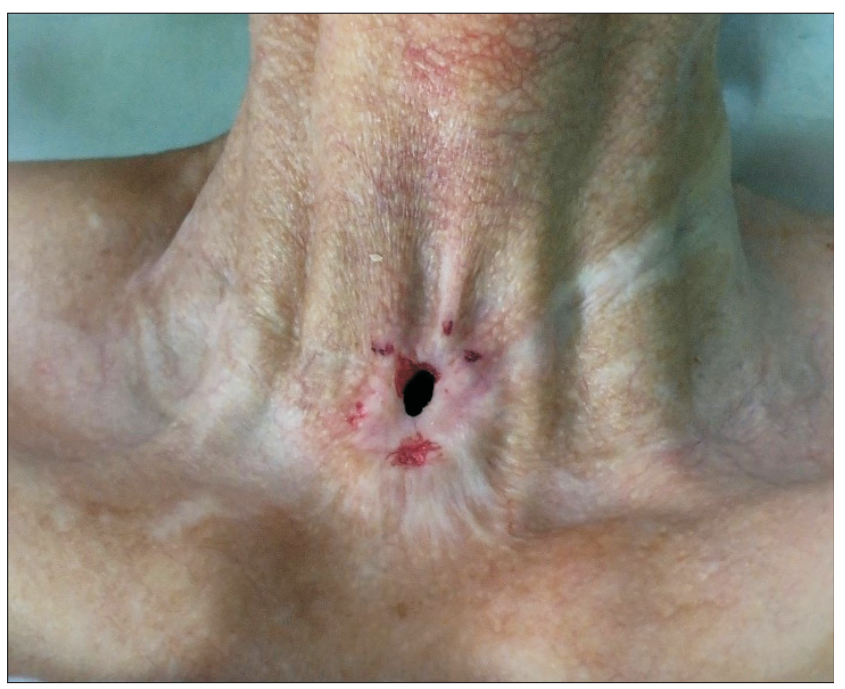

Fig. 4. 5-mm stoma narrowed by stenosis.

there was a risk of aspiration from bleeding, and tracheostomy was the sole airway. For this reason, we planned for stomaplasty after induction with fiberoptic scope-guided intubation, and replaced with a $7.0 \mathrm{~mm}$-ETT, operated by the general surgeon.

Induction was conducted in the presence of an otorhinolaryngologist as a standby in case of an emergency during intubation. Intubation failure can be fatal because mask ventilation is impossible due to a deformed airway. After confirming $100 \%$ preoxygenation by securing a highflow mask (20 L/min oxygen) around the tracheostomy stoma, $3 \mathrm{mg}$ of midazolam was administered, and intubation was attempted.

After checking the carina using a fiberoptic scope to prevent placement into any false passages or partial displacement, an ETT (ID 4.5 mm/OD $6.2 \mathrm{~mm}$ ) was inserted (Fig. 5). The tube was fixed at $9 \mathrm{~cm}$ from the skin and inflated $(7 \mathrm{cc}$ ). Ventilation sounds in both lungs were confirmed. Rocuronium $50 \mathrm{mg}$ was administered, and anesthesia was maintained with 1 to $1.5 \%$ minimum alveolar concentration of sevoflurane. Moreover, we used the volume control auto-flow mode (Persus A500, Drager). The setting was as follows: fraction of inspired oxygen, 0.6; fresh gas flow, $2.5 \mathrm{~L} / \mathrm{min}$; tidal volume, $7 \mathrm{~mL} / \mathrm{kg}$; respiratory rate, 14 breaths $/ \mathrm{min}$. The otolaryngologist performed stomaplasty while maintaining a high peak inspiratory pressure of $35 \mathrm{cmH}_{2} \mathrm{O}$, and the pressure was decreased to $21 \mathrm{cmH}_{2} \mathrm{O}$ after replacement using a 7.0-mm ETT (Fig. 6). Subsequently, Hartmann's operation

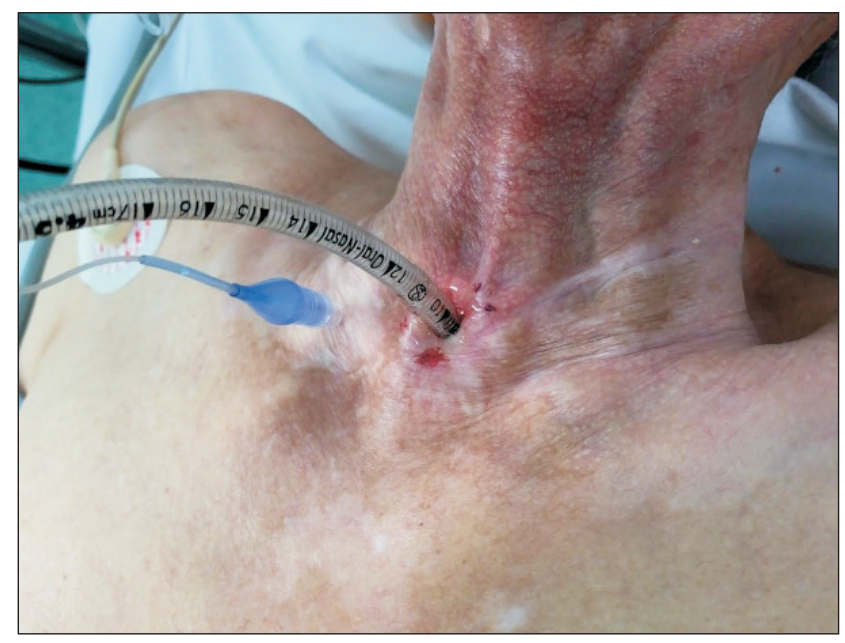

Fig. 5. Intubated with 4.5-mm endotracheal tube.

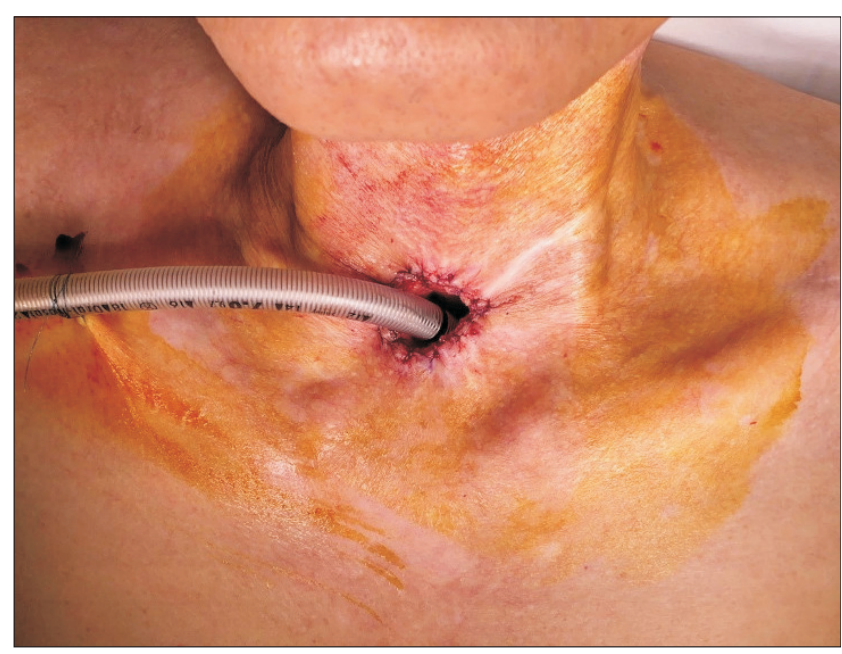

Fig. 6. Replaced with 7.0-mm endotracheal tube after tracheoplasty.

was performed without complications, and the patient was discharged to the intensive care unit under anesthesia after changing the \#8 T-tube (ID $7.6 \mathrm{~mm} / \mathrm{OD} 12.2 \mathrm{~mm}$, Shiley ${ }^{\mathrm{TM}}$, COVIDIEN $^{\mathrm{TM}}$ ). On the following day, the patient was weaned from mechanical ventilation and was able to breathe independently without complications.

An institutional review board approved the conduct of this case report (approval number: 2021-07-014).

\section{DISCUSSION}

With the advances in medical technology and average life expectancy, the types of difficult airways encountered by anesthesiologists in the operating room are becoming more 
diverse. This was a significant case involving the use of a neck breather, as the challenges associated with this difficult airway were different from those in patients with obesity, cervical spine injury, burn, or thermal injury. Patients with laryngeal cancer have different types of airways based on the surgical method of cordectomy and partial or total aryngectomy. The tracheostomy stoma might be the only airway, or two airways that might be available when the oropharyngeal airway is patent.

When intubating patients who are neck breathers, airway confusion due to different airway spaces depending on the surgery and complications can induce a major risk. The results of a survey conducted by Brenner et al. [2] among 254 practitioners in the US reported that the percentage of patients with a history of total laryngectomy who underwent oral intubation was $53.7 \%$, and the consequential mortality rate was $26 \%$. As such, it is important to accurately evaluate the airway of neck-breathing patients prior to intubation.

Patients who have undergone total laryngectomy have different types of airway structures based on the type of surgery and device insertion. During the preoperative evaluation, the anesthesiologist must consider the various accessories that can be used for the permanent stoma, such as the decannulation cap, speaking valve, and humidifier. Moreover, the area of the deformed airway, condition of the tracheal stoma, and associated complications should also be considered. McGrath et al. [3] emphasized that a bedside information card displaying a deformed airway is helpful in maintaining patient safety during emergency situations. A comprehensive plan must be created to outline whether intubation will be performed using the tracheostomy or the oropharynx, along with an alternative plan in the event of failed access [3,4]. Post-laryngectomy airway complications include insensate granulation tissue, stomal stenosis, and pharyngocutaneous fistula [5]. As various complications can interfere with intubation or the maintenance of ventilation, a multidisciplinary team approach, including a consultation with a head and neck specialist prior to surgery, and the evaluation of airway through laryngoscopy and CT imaging are essential.

Since mask ventilation is not possible with the nasopharynx, more attention should be paid to preoxygenation through self-respiration. This can also be achieved through the stoma using a small pediatric facemask, high flow, or laryngeal mask airway applied to the skin [3]. When performing intubation of the tracheostomy stoma, a fiberoptic scope should be used to facilitate the placement of an airway catheter or bougie, or to allow correct placement of a tracheal or tracheostomy tube [3].

After intubation, the position of the tube may be inaccurate because of the false passage or space deformation. Complications around the tracheostomy and tube malposition due to partial displacement can lead to life-threatening subcutaneous emphysema. Therefore, proper placement of the endotracheal tube using a fiberoptic scope can prevent one-lung ventilation or false passage. The use of waveform capnography enables the prompt diagnosis of tube blockage or displacement. The patency of the airway can also be easily checked by inserting it through a suction catheter [3].

Tracheomalacia, a long-term laryngectomy complication, may cause unexpected ventilation failure. In this case, emergency fiberoptic bronchoscopy may aid in the detection of expiratory central airway collapse and adjustment of the endotracheal tube position [6]. In case of short neck and obese patients, tracheostomy stoma intubation can be challenging. A case has been reported on the use of an ETT exchanger in an obese tracheostomy-maintaining patient with a short neck who had a false passage. Shoulder elevation and head extension postures can also be beneficial for safer and easier maneuvers [7,8].

For tracheostomy stoma intubation of neck-breather patients, a small-diameter ETT is recommended. Considering the lung protective strategy, it is suggested that a low tidal volume ( $6 \mathrm{~mL} / \mathrm{kg}$ predicted body weight) and plateau pressure of less than $30 \mathrm{cmH}_{2} \mathrm{O}$ be maintained. If airway patency is not an issue, an improvement in plateau pressure can be expected by using the pressure control or support mode of the mechanical ventilator. In our case, the pressure increase was due to the narrow tube diameter and not the patient's lung resistance. To resolve this complication and consequently relieve the patient's complaints of breathing discomfort, tracheoplasty was first performed [0].

In the case of patients with total laryngectomy, if intubation fails, mask ventilation may not be possible because of the current structure, which can easily develop into a cannot intubate, cannot oxygenate (CICO) state. Therefore, awake intubation is recommended, as the probability of a life-threatening emergency is exceedingly high when the 


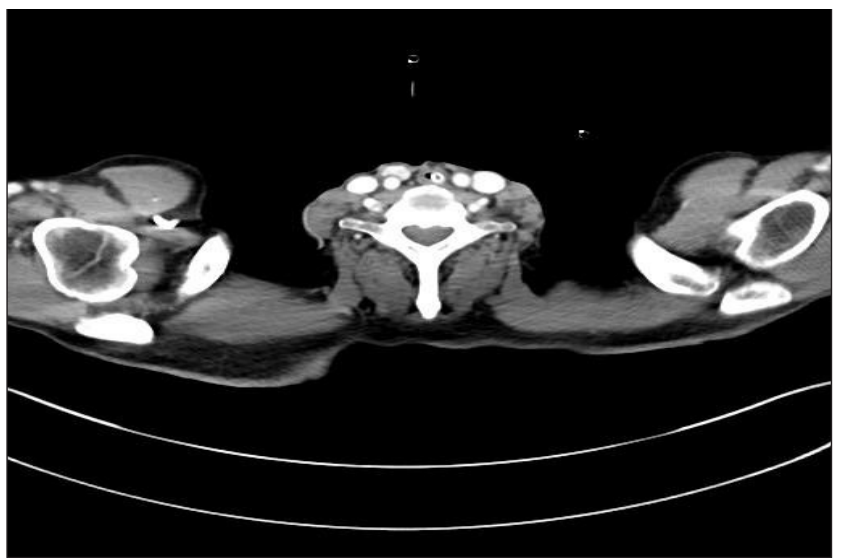

Fig. 7. Confirmed that the internal jugular vein is intact at the $\mathrm{C} 7$ level.

tracheostomy is blocked or lost [4]. All available personnel, resources, and plans should be prepared for these emergencies. Adequate preparations for a prompt front of the neck airway (FONA) or re-performing of the tracheostomy must be made. Venovenous extracorporeal membrane oxygenation (VV-ECMO) can also be considered as a safe measure to re-perform tracheostomy in patients who are expected to undergo respiratory decompensation. Depending on the total laryngectomy, there may be deformation of the internal jugular vein (IJV). In this case, intact IJV could be identified on CT (Fig. 7), and VV-ECMO could be considered [10]. Informed consent from the patient is necessary, considering the possibility of emergent situations. When CT imaging or fiberoptic scopes present challenges in securing the airway due to tracheal stenosis or granulation, general anesthesia can be considered after securing the airway using the Montgomery T-tube [11].

In conclusion, securing an airway for anesthesia in patients undergoing laryngectomy surgery is challenging. We successfully performed planned induction of anesthesia in a patient with deformed airway due to total laryngectomy and tracheostomy complications. Regarding the induction of anesthesia for a neck-breather patient, further research is needed in establishing guidelines or algorithms, including a collaboration with an otolaryngologist, understanding the airway space through imaging, using fiber optic scopes, and preparing for emergencies such as CICO.

\section{CONFLICT OF INTEREST}

No potential conflict of interest relevant to this article was reported.

\section{REFERENCES}

1. Korea Central Cancer Registry. 2018 registered cancer statistics [Internet]. Ilsan: National Cancer Center; 2021 [updated 2021 Jan 4; cited 2021 Aug 25]. Available from: https://ncc. re.kr/cancerStatsList.ncc?searchKey=total\&searchValue $=\&$ pageNum $=1$.

2. Brenner MJ, Cramer JD, McGrath BA, Balakrishnan K, Stepan KO, Pandian V, et al. Oral intubation attempts in patients with a laryngectomy: a significant safety threat. Otolaryngol Head Neck Surg 2021;164:1040-3.

3. McGrath BA, Bates L, Atkinson D, Moore JA; National Tracheostomy Safety Project. Multidisciplinary guidelines for the management of tracheostomy and laryngectomy airway emergencies. Anaesthesia 2012;67:1025-41.

4. Higgs A, McGrath BA, Goddard C, Rangasami J, Suntharalingam $\mathrm{G}$, Gale R, et al. Guidelines for the management of tracheal intubation in critically ill adults. Br J Anaesth 2018; 120:323-52.

5. Jackson C, Grigg C, Green M, Grigg R. Care of laryngectomy stomas in general practice. Aust J Gen Pract 2019;48:373-7.

6. Sonoda S, Sato K, Takagi Y, Sato Y, Murao F, Koide Y, et al. Undiagnosed tracheomalacia accompanied with accidental expiratory central airway collapse after tracheal intubation. Acute Med Surg 2021;8:e665.

7. Hwang SM, Jang JS, Yoo JI, Kwon HK, Lee SK, Lee JJ, et al. Difficult tracheostomy tube placement in an obese patient with a short neck -a case report-. Korean J Anesthesiol 2011; 60:434-6.

8. Sayoo C, Das AK, Das A, Rahman T, Das RJ, Das K. Redo tracheostomy: our experience, problems encountered and how to overcome them. Indian J Otolaryngol Head Neck Surg 2019;71:470-3.

9. Slutsky AS, Ranieri VM. Ventilator-induced lung injury. N Engl J Med 2013;369:2126-36.

10. Karim AS, Son AY, Suen R, Walter JM, Saine M, Kim SS, et al. Pre-intubation veno-venous extracorporeal membrane oxygenation in patients at risk for respiratory decompensation. J Extra Corpor Technol 2020;52:52-7.

11. Peng L, Wei W. Tube-in-tube airway management in a patient with montgomery T-tube in situ -a case report. Korean J Anesthesiol 2021;74:165-8. 